\title{
Methylsalicylate: a rotational spectroscopy study
}

Sonia Melandri*, Barbara Michela Giuliano, Assimo Maris, Laura B. Favero, Paolo Ottaviani, Biagio Velino and Walther Caminati

SUPPORTING INFORMATION AVAILABLE:

Complete ref. 17

Table 1s: Calculated geometries of the two stable rotamers of MS (I and II)

Table 2s: Experimental transition frequencies $(\mathrm{MHz})$ of $\mathrm{MS}$

Complete ref. 17:

Gaussian 03, Revision B.02, M. J. Frisch, G. W. Trucks, H. B. Schlegel, G. E. Scuseria, M. A. Robb, J. R. Cheeseman, J. A. Montgomery, Jr., T. Vreven, K. N. Kudin, J. C. Burant, J. M. Millam, S. S. Iyengar, J. Tomasi, V. Barone, B. Mennucci, M. Cossi, G. Scalmani, N. Rega, G. A. Petersson, H. Nakatsuji, M. Hada, M. Ehara, K. Toyota, R. Fukuda, J. Hasegawa, M. Ishida, T. Nakajima, Y. Honda, O. Kitao, H. Nakai, M. Klene, X. Li, J. E. Knox, H. P. Hratchian, J. B. Cross, C. Adamo, J. Jaramillo, R. Gomperts, R. E. Stratmann, O. Yazyev, A. J. Austin, R. Cammi, C. Pomelli, J. W. Ochterski, P. Y. Ayala, K. Morokuma, G. A. Voth, P. Salvador, J. J. Dannenberg, V. G. Zakrzewski, S. Dapprich, A. D. Daniels, M. C. Strain, O. Farkas, D. K. Malick, A. D. Rabuck, K. Raghavachari, J. B. Foresman, J. V. Ortiz, Q. Cui, A. G. Baboul, S. Clifford, J. Cioslowski, B. B. Stefanov, G. Liu, A. Liashenko, P. Piskorz, I. Komaromi, R. L. Martin, D. J. Fox, T. Keith, M. A. Al-Laham, C. Y. Peng, A. Nanayakkara, M. Challacombe, P. M. W. Gill, B. Johnson, W. Chen, M. W. Wong, C. Gonzalez, and J. A. Pople, Gaussian, Inc., Pittsburgh PA, 2003. 
Table 1s: Calculated geometries of the two stable rotamers of MS (I and II)

\begin{tabular}{|c|c|c|c|c|c|c|c|c|c|c|c|c|c|}
\hline \multicolumn{14}{|c|}{ B3LYP/6-31G(d,p) } \\
\hline \multicolumn{7}{|c|}{ II } & \multicolumn{7}{|c|}{ I } \\
\hline $\mathrm{C}$ & & & & & & & $\mathrm{C}$ & & & & & & \\
\hline $\mathrm{C}$ & 1 & 1.40487 & & & & & $\mathrm{C}$ & 1 & 1.40439 & & & & \\
\hline $\mathrm{C}$ & 2 & 1.38659 & 1 & 120.219 & & & $\mathrm{C}$ & 2 & 1.38681 & 1 & 120.621 & & \\
\hline $\mathrm{C}$ & 3 & 1.40397 & 2 & 121.004 & 1 & 0.000 & $\mathrm{C}$ & 3 & 1.40232 & 2 & 120.697 & 1 & 0.000 \\
\hline $\mathrm{C}$ & 4 & 1.38530 & 3 & 119.301 & 2 & 0.000 & $\mathrm{C}$ & 4 & 1.38470 & 3 & 119.087 & 2 & 0.000 \\
\hline $\mathrm{C}$ & 5 & 1.40831 & 4 & 120.897 & 3 & 0.000 & $\mathrm{C}$ & 5 & 1.40887 & 4 & 121.489 & 3 & 0.000 \\
\hline $\mathrm{H}$ & 2 & 1.08457 & 1 & 118.067 & 3 & 180.000 & $\mathrm{H}$ & 2 & 1.08467 & 1 & 117.794 & 3 & 180.000 \\
\hline $\mathrm{H}$ & 3 & 1.08642 & 2 & 119.245 & 4 & 180.000 & $\mathrm{H}$ & 3 & 1.08634 & 2 & 119.306 & 4 & 180.000 \\
\hline $\mathrm{H}$ & 4 & 1.08484 & 3 & 120.376 & 5 & 180.000 & $\mathrm{H}$ & 4 & 1.08483 & 3 & 120.536 & 5 & 180.000 \\
\hline $\mathrm{H}$ & 5 & 1.08395 & 4 & 120.640 & 3 & 180.000 & $\mathrm{H}$ & 5 & 1.08458 & 4 & 121.229 & 3 & 180.000 \\
\hline $\mathrm{C}$ & 6 & 1.46903 & 5 & 122.051 & 4 & 180.000 & $\mathrm{C}$ & 6 & 1.47997 & 5 & 116.232 & 4 & 180.000 \\
\hline $\mathrm{O}$ & 1 & 1.34232 & 2 & 117.993 & 3 & 180.000 & $\mathrm{O}$ & 1 & 1.35039 & 2 & 116.398 & 3 & 180.000 \\
\hline $\mathrm{H}$ & 12 & 0.98723 & 1 & 106.980 & 2 & 180.000 & $\mathrm{H}$ & 12 & 0.97453 & 1 & 108.251 & 2 & 180.000 \\
\hline $\mathrm{O}$ & 11 & 1.23359 & 6 & 124.060 & 5 & 180.000 & $\mathrm{O}$ & 11 & 1.21279 & 6 & 125.679 & 5 & 0.000 \\
\hline $\mathrm{O}$ & 11 & 1.34382 & 14 & 121.689 & 6 & 180.000 & $\mathrm{O}$ & 11 & 1.37479 & 14 & 121.195 & 6 & 180.000 \\
\hline $\mathrm{C}$ & 15 & 1.43819 & 11 & 115.772 & 14 & 0.000 & $\mathrm{C}$ & 15 & 1.44003 & 11 & 115.590 & 14 & 0.000 \\
\hline $\mathrm{H}$ & 16 & 1.08941 & 15 & 105.406 & 11 & 180.000 & $\mathrm{H}$ & 16 & 1.08952 & 15 & 105.644 & 11 & 180.000 \\
\hline $\mathrm{H}$ & 16 & 1.09233 & 15 & 110.590 & 17 & 119.619 & $\mathrm{H}$ & 16 & 1.09174 & 15 & 110.309 & 17 & 119.715 \\
\hline $\mathrm{H}$ & 16 & 1.09233 & 15 & 110.590 & 17 & 240.381 & $\mathrm{H}$ & 16 & 1.09174 & 15 & 110.309 & 17 & 240.285 \\
\hline \multicolumn{14}{|c|}{ MP2/6-311++G(d,p) } \\
\hline \multicolumn{7}{|c|}{ II } & \multicolumn{7}{|c|}{ I } \\
\hline $\mathrm{C}$ & & & & & & & $\mathrm{C}$ & & & & & & \\
\hline $\mathrm{C}$ & 1 & 1.40464 & & & & & $\mathrm{C}$ & 1 & 1.40470 & & & & \\
\hline $\mathrm{C}$ & 2 & 1.39181 & 1 & 120.508 & & & $\mathrm{C}$ & 2 & 1.39161 & 1 & 120.989 & & \\
\hline $\mathrm{C}$ & 3 & 1.40477 & 2 & 120.475 & 1 & 2.509 & $\mathrm{C}$ & 3 & 1.40372 & 2 & 120.203 & 1 & 0.000 \\
\hline $\mathrm{C}$ & 4 & 1.39103 & 3 & 119.518 & 2 & -2.51 & $\mathrm{C}$ & 4 & 1.39015 & 3 & 119.334 & 2 & 0.000 \\
\hline $\mathrm{C}$ & 5 & 1.40942 & 4 & 120.597 & 3 & 3.238 & $\mathrm{C}$ & 5 & 1.40986 & 4 & 121.203 & 3 & 0.000 \\
\hline $\mathrm{H}$ & 2 & 1.08601 & 1 & 118.008 & 3 & 182.454 & $\mathrm{H}$ & 2 & 1.08612 & 1 & 117.688 & 3 & 180.000 \\
\hline
\end{tabular}




\begin{tabular}{|ccccccc|ccccccc}
$\mathrm{H}$ & 3 & 1.08684 & 2 & 119.393 & 4 & 177.878 & $\mathrm{H}$ & 3 & 1.08671 & 2 & 119.489 & 4 & 180.000 \\
$\mathrm{H}$ & 4 & 1.08568 & 3 & 120.366 & 5 & 182.675 & $\mathrm{H}$ & 4 & 1.08566 & 3 & 120.530 & 5 & 180.000 \\
$\mathrm{H}$ & 5 & 1.08481 & 4 & 120.683 & 3 & 180.262 & $\mathrm{H}$ & 5 & 1.08572 & 4 & 121.076 & 3 & 180.000 \\
$\mathrm{C}$ & 6 & 1.47716 & 5 & 121.402 & 4 & 181.254 & $\mathrm{C}$ & 6 & 1.48697 & 5 & 115.964 & 4 & 180.000 \\
$\mathrm{O}$ & 1 & 1.35049 & 6 & 123.407 & 11 & -3.73 & $\mathrm{O}$ & 1 & 1.35663 & 2 & 115.988 & 3 & 180.000 \\
$\mathrm{O}$ & 11 & 1.22838 & 6 & 123.997 & 5 & 178.075 & $\mathrm{H}$ & 12 & 0.96975 & 1 & 107.302 & 2 & 180.000 \\
$\mathrm{H}$ & 13 & 1.75775 & 11 & 100.220 & 6 & -1.56 & $\mathrm{O}$ & 11 & 1.21046 & 6 & 125.584 & 5 & 0.000 \\
$\mathrm{O}$ & 11 & 1.34070 & 6 & 113.628 & 5 & -2.49 & $\mathrm{O}$ & 11 & 1.36971 & 14 & 121.910 & 6 & 180.000 \\
$\mathrm{C}$ & 15 & 1.43903 & 11 & 114.630 & 6 & 181.082 & $\mathrm{C}$ & 15 & 1.44021 & 11 & 114.704 & 14 & 0.000 \\
$\mathrm{H}$ & 16 & 1.08812 & 15 & 105.045 & 11 & 179.709 & $\mathrm{H}$ & 16 & 1.08850 & 15 & 105.334 & 11 & 180.000 \\
$\mathrm{H}$ & 16 & 1.09100 & 15 & 110.333 & 11 & 299.164 & $\mathrm{H}$ & 16 & 1.09057 & 15 & 110.078 & 17 & 119.530 \\
$\mathrm{H}$ & 16 & 1.09104 & 15 & 110.280 & 11 & 60.243 & $\mathrm{H}$ & 16 & 1.09057 & 15 & 110.078 & 17 & 240.470 \\
\hline \hline
\end{tabular}


Table 2s. Experimental transition frequencies (MHz) of MS

\begin{tabular}{|c|c|c|c|c|c|c|c|c|}
\hline & \multicolumn{4}{|c|}{$\mathrm{MS}(\mathrm{OH})$} & \multicolumn{4}{|c|}{$\mathrm{MS}(\mathrm{OD})$} \\
\hline$J^{\prime}\left(K^{\prime}{ }_{a}, K^{\prime}{ }_{c}\right) \leftarrow J^{\prime \prime}\left(K^{\prime \prime}{ }_{a}, K^{\prime \prime}\right)$ & $\mathrm{A}$ & & $E^{a}$ & & A & & $\mathrm{E}^{\mathrm{a}}$ & \\
\hline $15(14)-14(13)^{b}$ & 60739.39 & -0.02 & $\begin{array}{l}60735.35 \\
60740.13\end{array}$ & $\begin{array}{r}-0.02 \\
0.05\end{array}$ & & & & \\
\hline $15(15)-14(14)^{b}$ & 63632.15 & 0.00 & $\begin{array}{r}63632.71 \\
63628\end{array}$ & $\begin{array}{r}0.03 \\
-0.02\end{array}$ & 62323.17 & 0.00 & $\begin{array}{l}62323.78 \\
62319.16\end{array}$ & $\begin{array}{l}-0.01 \\
-0.01\end{array}$ \\
\hline $16(14)-15(13)^{b}$ & 62184.66 & -0.05 & $\begin{array}{l}62185.41 \\
62180.68\end{array}$ & $\begin{array}{r}-0.00 \\
0.01\end{array}$ & 60959.48 & 0.02 & $\begin{array}{l}60960.14 \\
60955.52\end{array}$ & $\begin{array}{l}-0.07 \\
-0.03\end{array}$ \\
\hline $16(15)-15(14)^{b}$ & 65077.7 & -0.01 & $\begin{array}{l}65078.26 \\
65073.66\end{array}$ & $\begin{array}{r}-0.02 \\
0.06\end{array}$ & 63765.52 & -0.00 & $\begin{array}{l}63766.13 \\
63761.54\end{array}$ & $\begin{array}{r}-0.02 \\
0.02\end{array}$ \\
\hline $16(16)-15(15)^{b}$ & 67970.38 & -0.00 & $\begin{array}{l}67966.14 \\
67970.72\end{array}$ & $\begin{array}{l}-0.03 \\
-0.07\end{array}$ & 66571.23 & 0.00 & $\begin{array}{l}66567.14 \\
66571.71\end{array}$ & $\begin{array}{r}-0.01 \\
0.00\end{array}$ \\
\hline $17(13)-16(12)^{b}$ & 60734.84 & 0.02 & $\begin{array}{l}60730.92 \\
60735.71\end{array}$ & $\begin{array}{l}0.04 \\
0.03\end{array}$ & & & & \\
\hline $17(14)-16(13)^{b}$ & 63629.56 & 0.03 & $\begin{array}{l}63625.48 \\
63630.25\end{array}$ & $\begin{array}{r}-0.02 \\
0.01\end{array}$ & 62401.02 & 0.00 & $\begin{array}{l}62397.16 \\
62401.75\end{array}$ & $\begin{array}{r}0.05 \\
-0.02\end{array}$ \\
\hline $17(15)-16(14)^{b}$ & 66523.11 & 0.05 & $\begin{array}{l}66519.01 \\
66523.64\end{array}$ & $\begin{array}{l}0.08 \\
0.03\end{array}$ & 65207.62 & -0.01 & 65203.64 & 0.00 \\
\hline $17(16)-16(15)^{b}$ & 69415.98 & 0.03 & $\begin{array}{l}69411.72 \\
69416.41\end{array}$ & $\begin{array}{r}-0.02 \\
0.03\end{array}$ & 68013.6 & 0.02 & $\begin{array}{r}68009.45 \\
68014.1\end{array}$ & $\begin{array}{r}-0.04 \\
0.04\end{array}$ \\
\hline $17(17)-16(16)^{b}$ & 72308.5 & -0.05 & $\begin{array}{l}72304.35 \\
72308.76\end{array}$ & $\begin{array}{r}0.09 \\
-0.06\end{array}$ & 70819.18 & -0.03 & $\begin{array}{l}70815.04 \\
70819.62\end{array}$ & $\begin{array}{r}-0.01 \\
0.06\end{array}$ \\
\hline $18(13)-17(12)^{b}$ & 62177.55 & -0.01 & $\begin{array}{l}62173.62 \\
62178.39\end{array}$ & $\begin{array}{r}0.01 \\
-0.03\end{array}$ & 61032.46 & -0.03 & $\begin{array}{l}61028.63 \\
61033.36\end{array}$ & $\begin{array}{l}-0.03 \\
-0.03\end{array}$ \\
\hline $18(14)-17(13)^{b}$ & 65073.66 & -0.01 & $\begin{array}{l}65069.66 \\
65074.39\end{array}$ & $\begin{array}{l}0.03 \\
0.01\end{array}$ & 63841.84 & -0.00 & 63837.91 & -0.02 \\
\hline $18(15)-17(14)^{b}$ & 67967.96 & -0.03 & $\begin{array}{l}67963.78 \\
67968.53\end{array}$ & $\begin{array}{l}-0.08 \\
-0.01\end{array}$ & 66649.32 & 0.02 & 66645.3 & -0.01 \\
\hline $18(16)-17(15)^{b}$ & 70861.33 & 0.01 & $\begin{array}{l}70857.09 \\
70861.75\end{array}$ & $\begin{array}{r}-0.01 \\
0.01\end{array}$ & 69455.8 & 0.08 & $\begin{array}{l}69451.65 \\
69456.23\end{array}$ & $\begin{array}{l}0.01 \\
0.03\end{array}$ \\
\hline $18(17)-17(16)^{b}$ & 73754.25 & 0.13 & $\begin{array}{l}73749.79 \\
73754.42\end{array}$ & $\begin{array}{r}-0.04 \\
0.03\end{array}$ & 72261.53 & -0.02 & $\begin{array}{l}72257.35 \\
72261.89\end{array}$ & $\begin{array}{l}-0.05 \\
-0.01\end{array}$ \\
\hline $18(18)-17(17)^{b}$ & & & & & 75067.15 & 0.04 & $\begin{array}{l}75062.82 \\
75067.41\end{array}$ & $\begin{array}{r}-0.06 \\
0.09\end{array}$ \\
\hline $19(12)-18(11)^{b}$ & 60716.39 & -0.02 & $\begin{array}{l}60712.59 \\
60717.39\end{array}$ & $\begin{array}{r}0.03 \\
-0.03\end{array}$ & & & & \\
\hline $19(13)-18(12)^{b}$ & 63618.84 & -0.02 & $\begin{array}{l}63614.91 \\
63619.74\end{array}$ & $\begin{array}{l}0.00 \\
0.03\end{array}$ & 62466.53 & 0.07 & 62470.25 & -0.04 \\
\hline $19(14)-18(13)^{b}$ & 66516.84 & -0.02 & $\begin{array}{l}66512.9 \\
66517.6\end{array}$ & $\begin{array}{l}0.08 \\
0.03\end{array}$ & 65281.73 & 0.05 & $\begin{array}{l}65277.77 \\
65282.46\end{array}$ & $\begin{array}{l}0.01 \\
0.02\end{array}$ \\
\hline $19(15)-18(14)^{b}$ & 69412.34 & 0.01 & $\begin{array}{l}69408.27 \\
69412.93\end{array}$ & $\begin{array}{l}0.07 \\
0.03\end{array}$ & 68090.31 & -0.05 & $\begin{array}{l}68086.33 \\
68090.92\end{array}$ & $\begin{array}{l}-0.03 \\
-0.06\end{array}$ \\
\hline $19(16)-18(15)^{b}$ & 72306.35 & 0.01 & $\begin{array}{l}72302.13 \\
72306.71\end{array}$ & $\begin{array}{r}0.06 \\
-0.04\end{array}$ & 70897.49 & 0.00 & $\begin{array}{l}70893.43 \\
70897.99\end{array}$ & $\begin{array}{l}0.02 \\
0.02\end{array}$ \\
\hline $19(17)-18(16)^{\mathrm{b}}$ & 75199.51 & 0.01 & $\begin{array}{l}75195.08 \\
75199.73\end{array}$ & $\begin{array}{l}-0.13 \\
-0.04\end{array}$ & 73703.73 & 0.01 & $\begin{array}{l}73699.49 \\
73703.99\end{array}$ & $\begin{array}{l}-0.07 \\
-0.08\end{array}$ \\
\hline $20(12)-19(11)^{b}$ & 62151.84 & -0.01 & $\begin{array}{l}62147.99 \\
62152.88\end{array}$ & $\begin{array}{l}0.01 \\
0.02\end{array}$ & 61085.7 & -0.04 & $\begin{array}{l}61082.04 \\
61086.89\end{array}$ & $\begin{array}{l}0.05 \\
0.11\end{array}$ \\
\hline $20(13)-19(12)^{b}$ & 65058.33 & -0.01 & $\begin{array}{l}65054.36 \\
65059.15\end{array}$ & $\begin{array}{l}-0.02 \\
-0.05\end{array}$ & 63906.17 & -0.00 & $\begin{array}{l}63902.32 \\
63907.08\end{array}$ & $\begin{array}{r}-0.02 \\
0.01\end{array}$ \\
\hline $20(14)-19(13)^{b}$ & 67958.79 & -0.06 & 67954.78 & -0.02 & 66720.23 & 0.00 & 66716.36 & 0.06 \\
\hline
\end{tabular}




\begin{tabular}{|c|c|c|c|c|c|c|c|c|}
\hline \multirow{3}{*}{$20(15)-19(14)^{b}$} & & & 67959.51 & -0.05 & & & & \\
\hline & 70855.92 & 0.04 & 70851.75 & 0.00 & 69530.56 & -0.00 & 69526.63 & 0.06 \\
\hline & & & 70856.46 & 0.02 & & & 69531.17 & -0.02 \\
\hline \multirow[t]{2}{*}{$20(16)-19(15)^{b}$} & 73750.81 & -0.03 & 73746.6 & -0.03 & 72338.71 & 0.00 & 72334.64 & 0.06 \\
\hline & & & 73751.21 & -0.04 & & & 72339.18 & -0.01 \\
\hline \multirow[t]{2}{*}{$20(17)-19(16)^{b}$} & & & & & 75145.51 & -0.04 & 75141.47 & 0.07 \\
\hline & & & & & & & 75145.85 & -0.05 \\
\hline \multirow[t]{2}{*}{$22(11)-21(10)^{b}$} & 62076.45 & 0.07 & 62072.66 & -0.00 & & & & \\
\hline & & & 62077.68 & 0.03 & & & & \\
\hline \multirow[t]{2}{*}{$21(12)-20(11)^{b}$} & 63583.98 & -0.02 & 63580.11 & -0.01 & 62513.76 & -0.03 & 62509.99 & -0.04 \\
\hline & & & 63585.04 & 0.03 & & & & \\
\hline \multirow{2}{*}{$21(11)-20(10)^{b}$} & 60658.56 & 0.02 & 60654.78 & 0.00 & & & & \\
\hline & & & 60659.74 & -0.00 & & & & \\
\hline $23(10,14)-22(9,13)$ & 60493.04 & 0.01 & 60493.39 & -0.03 & & & & \\
\hline $23(10,13)-22(9,14)$ & 60500.89 & 0.04 & 60498.1 & 0.04 & & & & \\
\hline \multirow[t]{2}{*}{$24(10)-23(9)^{b}$} & 61864.18 & -0.02 & 61870.34 & -0.01 & & & & \\
\hline & & & 61873.07 & 0.02 & & & & \\
\hline $25(9,17)-24(8,16)$ & 59829.71 & -0.08 & 59821.15 & -0.04 & & & & \\
\hline $25(9,16)-24(8,17)$ & 60240.18 & 0.07 & 60246.5 & -0.00 & & & & \\
\hline \multirow[t]{2}{*}{$23(11)-22(10)^{b}$} & & & 63483.7 & 0.08 & & & & \\
\hline & & & 63488.64 & -0.00 & & & & \\
\hline $25(10,16)-24(9,15)$ & 63214.8 & 0.03 & 63234.66 & 0.03 & & & & \\
\hline $25(10,15)-24(9,16)$ & 63251.8 & -0.02 & 63229.64 & 0.10 & & & & \\
\hline $26(9,17)-25(8,18)$ & 61663.4 & -0.09 & 61666.39 & -0.07 & & & & \\
\hline $26(9,18)-25(8,17)$ & 60917.68 & 0.06 & 60912.44 & -0.03 & & & & \\
\hline $27(9,19)-26(8,18)$ & 61852.42 & -0.03 & 61849.11 & 0.07 & & & & \\
\hline $27(9,18)-26(8,19)$ & 63153.23 & 0.03 & 63154.34 & -0.01 & & & & \\
\hline
\end{tabular}

${ }^{a}$ For lines which originate from degenerate levels there are two lines with E symmetry $\left(\mathrm{E}^{+}, \mathrm{E}^{-}\right) .{ }^{16}$

${ }^{b}$ Transitions coalesced due to near prolate degeneracy. Only $K_{a}$ is given. 